\title{
Technology and Investigation of Ohmic Contacts to Thermoelectric Materials
}

\author{
Y.I. Shtern*, R.E. Mironov, M.Y. Shtern, A.A. Sherchenkov and M.S. Rogachev \\ National Research University of Electronic Technology, \\ Russian Federation, Moscow, Zelenograd, Shokin Square, Building 1
}

\begin{abstract}
Technology is developed, materials and regimes of the fabrication of ohmic contacts to the effective thermoelectric materials $\mathrm{Bi}_{2} \mathrm{Te}_{2.8} \mathrm{Se}_{0.2}$ ( $n$-type) and $\mathrm{Bi}_{0.5} \mathrm{Sb}_{1.5} \mathrm{Te}_{3}$ (p-type) are determined. Ohmic contacts were obtained by the vacuum deposition of nickel. Factors determining adhesion strength and resistivity of fabricated contacts are determined. Process of surface preparation of the thermoelectric materials before the ohmic contact deposition is optimized during the technology development. The use of electrochemical polishing, ultrasound treatment, finish cleaning in toluene and isopropyl alcohol vapor, and annealing in vacuum allowed achieving stable results in the formation of contacts. It was shown that contacts fabricated using of electron-beam evaporation of nickel possess maximum adhesion strength of $18-19 \mathrm{~N} / \mathrm{mm}^{2}$. It was found that high adhesion is caused by the existence of transition layer in the metal-thermoelectric material contact range, formed due to the interaction of metal with the components of thermoelectric material. Proposed technology allows obtaining ohmic contacts with the resistance of the unit area not exceeding $10^{-10} \mathrm{Ohm} \mathrm{m}^{2}$.
\end{abstract}

DOI: 10.12693/APhysPolA.129.785

PACS/topics: $68.35 . \mathrm{Np}$

\section{Introduction}

One of the main problems connected with the increase of the efficiency of thermoelectric devices is the formation of ohmic contacts to the thermo-elements with high adhesion strength. Contacts to the thermo-elements on the basis of thermoelectric material bismuth telluride must have resistance not more than $10^{-9} \mathrm{Ohm} \mathrm{m}^{2}$, and adhesion strength not less than $8 \mathrm{~N} / \mathrm{mm}^{2}[1,2]$.

Ohmic contacts were fabricated by the vacuum deposition of nickel on the thermoelectric materials $\mathrm{Bi}_{2} \mathrm{Te}_{2.8} \mathrm{Se}_{0.2}$ and $\mathrm{Bi}_{0.5} \mathrm{Sb}_{1.5} \mathrm{Te}_{3}$ having $n$ - and $p$-types, respectively. These materials were fabricated by the vertical zone melting and had high thermoelectric figure of merit $Z=3.2 \times 10^{-3} \mathrm{~K}^{-1}$.

Control of electrical parameters of ohmic contacts was carried on using the developed methods and measurement apparatus equipped with high-quality devices with a resolution of $10^{-8} \mathrm{~V}$ and $10^{-6} \mathrm{Ohm}$ (multimeter Keithley-2001, precision bridge Instek LCR-819). Estimation of the contact resistance was carried out using method based on the comparison of the thermo-element resistance, calculated using the known conductivity of thermoelectric materials and the experimental data.

Adhesion strength qualitatively characterizes true adhesion. Method of uniform normal tear allows the most accurate determination of the adhesion strength [3]. This method was realized with the use of facility Force Gauge PCE-FM50, which has a relative error not exceeding $1 \%$.

*corresponding author; e-mail: hptt@miee.ru

\section{Preparation of thermal elements for the formation of ohmic contacts}

The Condition of the surface, on which the contact metal film is deposited, is crucial for the adhesion. Surface condition also has sufficient influence on the contact resistance [4]. In this connection, serious attention was devoted to the preparation of thermoelectric material surface during the formation of ohmic contacts [5]. At the first stage polishing electrochemical etching of sample surface is carried out. Uniform material removal on the entire surface providing minimal roughness is necessary for the polishing etching. Polishing etching promotes removal of the damaged surface layer of semiconductor, resulting from mechanical processing, and also cleans surface from the oxides and adsorbed contaminations.

Aqueous solutions of alkalis were used as solvents of the polishing electrolyte. Introduction of complex forming tartaric acid into the electrolyte increases solubility of the reaction products. Good results were obtained by using for the polishing electrochemical etching of the electrolyte with the following ingredients. For the $\mathrm{Bi}_{0.5} \mathrm{Sb}_{1.5} \mathrm{Te}_{3}$ ( $p$ type): $\mathrm{KOH}-(85-95) \mathrm{g} / \mathrm{l} ; \mathrm{H}_{2} \mathrm{C}_{4} \mathrm{H}_{4} \mathrm{O}_{6}-(60-70) \mathrm{g} / \mathrm{l}$; deionized water. For $\mathrm{Bi}_{2} \mathrm{Te}_{2.8} \mathrm{Se}_{0.2}$ (n-type): $\mathrm{NaOH}-$ (70-80) g/l; $\mathrm{H}_{2} \mathrm{C}_{4} \mathrm{H}_{4} \mathrm{O}_{6}-(60-70) \mathrm{g} / \mathrm{l}$; deionized water. Current density during the processing of the $p$ type material was $20 \mathrm{~A} / \mathrm{dm}^{2}$, and the processing time was $6 \mathrm{~min}$. For the samples of $n$-type the current density was $22 \mathrm{~A} / \mathrm{dm}^{2}$, and processing time was $8 \mathrm{~min}$.

It is impossible to entirely remove reaction products from the sample surface during the electrochemical etching. Ultrasonic $(50 \mathrm{kHz})$ processing of the sample in the suspension of the abrasive powder and aqueous solutions of tartaric acid $(100 \mathrm{~g} / \mathrm{l})$ is used for the intensification of surface cleaning process after the electrochemical etching. 
Finishing cleaning of the sample surface immediately before the metal contact deposition includes triple boiling of the sample in the toluene for 3-4 minutes with following processing in isopropyl alcohol vapor.

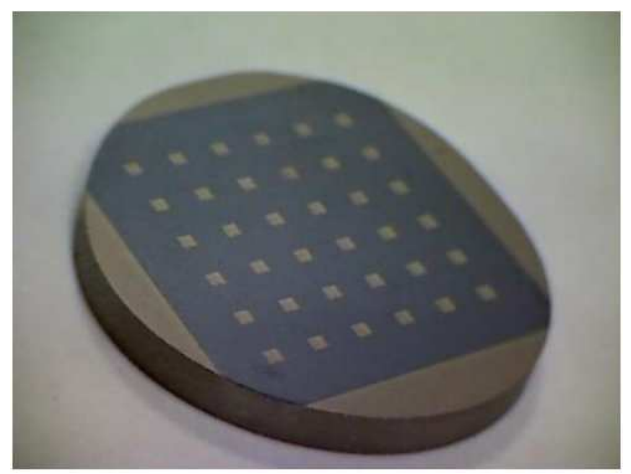

Fig. 1. The tablet of investigated material with contact pads.

The samples for the investigation of adhesion strength of metal films were fabricated as follows. Nickel films were deposited on both sides of pretreated surfaces of the tablets produced from the thermoelectric material. Then, contact pads with the sizes of $1 \mathrm{~mm}^{2}$ were fabricated by photolithography (Fig. 1). Nickel wire terminals were soldered to them, and adhesion strength of contact pads was measured by the method of direct tear.

\section{Fabrication and investigation of ohmic contacts to the thermoelectric materials}

During the development of the technology of forming contacts to the thermoelectric materials vacuum deposition of metal films was used, and investigations of adhesion strength of contacts were carried out. Selection of the material for the contact was based on the following considerations. Among the traditionally applied in thin film technology metals, the preference for the contact formation was given to nickel due to the complex of properties (resistivity, oxidizing ability, manufacturability of connection, diffusion ability and price). To provide extremely low contact resistance it is better not to use adhesion sublayer during the formation of the metallization to the thermoelectric materials. Investigations of nickel contacts show that this metal has high adhesion to the thermoelectric material. In addition, nickel possesses good protective properties, which prevent diffusion in the semiconductor of the solder components and copper, used for the formation of connections in a thermo-element. This prevents degradation of thermo-element parameters during its exploitation. Experiments with the fabrication of metal films by the thermal vacuum evaporation, using the resistive heating of material, allowed a slight increase of the film adhesion to the thermoelectric material (up to $6 \mathrm{~N} / \mathrm{mm}^{2}$ ) in comparison with the previously obtained results, which were using the same method for the formation of thermoelectric material metallization.
The use of ion-plasma method for metallization of thermoelectric materials allowed to sufficiently increase the adhesion of contacts in comparison with that of the contacts obtained by thermal vacuum evaporation. Adhesion strength for such contacts is equal to 10.5 and $10 \mathrm{~N} / \mathrm{mm}^{2}$ for $n$ - and $p$-type materials, respectively.

Metal film deposition with high deposition rate is possible by the electron-beam evaporation. Nickel films were deposited at the pressure of $10^{-3} \mathrm{~Pa}$. Thermalvacuum annealing of sample at temperatures of 420 $490 \mathrm{~K}$ for $15 \mathrm{~min}$ was used before the metal deposition. Deposition rates of nickel films were in the range of $10-40 \AA / \mathrm{s}$. Nickel films deposited by three methods (vacuum-thermal evaporation, ion-plasma method and electron-beam evaporation) were investigated by massspectrometry. Scanning of the film thickness was carried out by the nickel removal in the oxygen plasma on the depth of $10^{-7} \mathrm{~m}$. Investigations showed that nickel films contain tellurium, bismuth, antimony, concentration of which increases with the approach to the metal-thermoelectric material boundary. In addition, it was found that nickel work function changes in the contact range. So, it can be assumed that compositions of nickel with the thermoelectric material components are formed on the contact boundary. Calculations of the free energy of reactions allowed to estimate thermodynamically most probable reaction, which can occur during the deposition of nickel on thermoelectric material. Gibbs free energies of reactions were calculated considering that $\Delta C_{P} \neq f(T)$, using the equation

$$
\Delta G_{T}=\Delta H_{298}^{0}-T \Delta S_{298}^{0}-T \Delta C_{P 298} F,
$$

where $\Delta G_{T}$ is Gibbs free energy, kcal $/ \mathrm{mol} ; \Delta H_{298}^{0}$ is reaction enthalpy, $\mathrm{kcal} / \mathrm{mol} ; \Delta S_{298}^{0}$ is variation of reaction enthalpy, $\mathrm{kcal} / \mathrm{mol} \mathrm{K} ; \Delta C_{P 298}$ is variation of isobaric heat capacity of the reaction $\mathrm{kcal} / \mathrm{mol} \mathrm{K}$; $T$ is temperature, $\mathrm{K}$; $F$ is parameter determined by the equation

$$
F=\left(\ln \frac{T}{298}+\frac{298}{T}-1\right) \text {. }
$$

Calculations have shown that formation of compounds between the nickel and selenium is thermodynamically impossible. Most probable are the reactions with formation of nickel telluride and nickel antimonide.

Comparison of results obtained by three deposition methods have shown that films deposited by the electron beam evaporation have the highest adhesion. Investigations have also shown that optimal regimes lead to formation of contacts with the adhesion strengths of 19.5 and $18.7 \mathrm{~N} / \mathrm{mm}^{2}$ for $n$ - and $p$-types materials, respectively. Dependences of adhesion strengths of nickel contacts on the film thickness $h$ and process temperature $T_{p}$ are presented in Fig. 2

When analyzing the dependence of adhesion strength of nickel film on its thickness (Fig. 2a) it must be taken into account that the roughness of the initial surface of samples was $(1-1.2) \times 10^{-7} \mathrm{~m}$. In this connection adhesion strength of the films with the thicknesses less than $1.5 \times 10^{-7} \mathrm{~m}$ sharply drops. Increase of the nickel thickness above $1 \times 10^{-6} \mathrm{~m}$ is useless, because it leads to the 

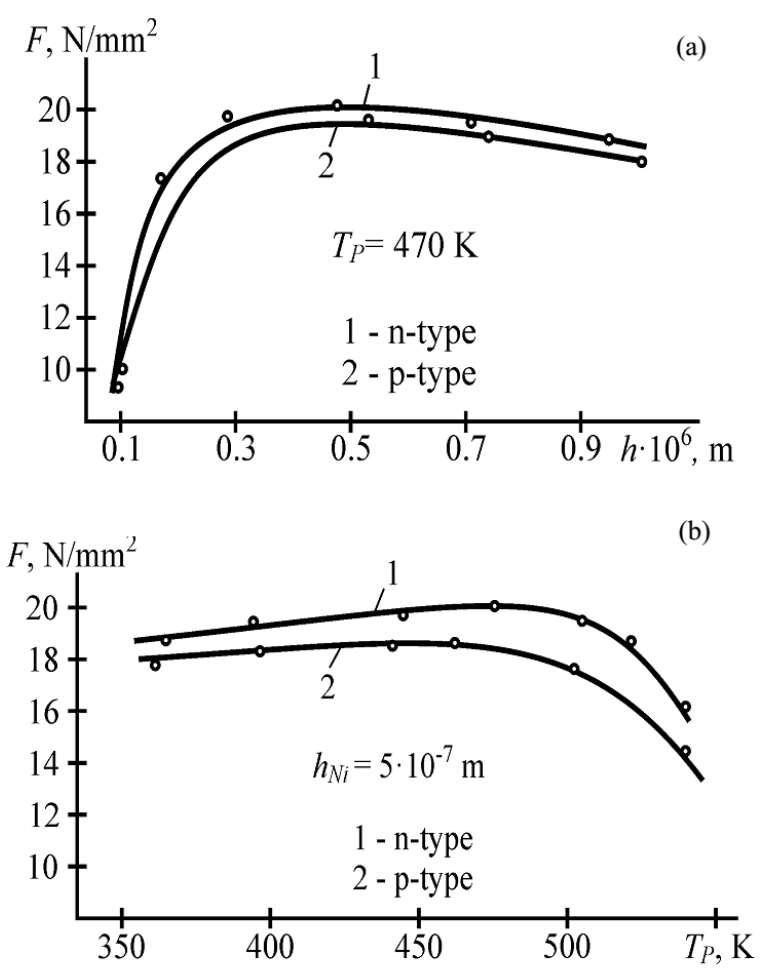

Fig. 2. Dependences of the strength of the adhesion of nickel contacts to the thermoelectric materials $\mathrm{Bi}_{2} \mathrm{Te}_{2.8} \mathrm{Se}_{0.2}$ ( $n$-type) and $\mathrm{Bi}_{0.5} \mathrm{Sb}_{1.5} \mathrm{Te}_{3}$ (p-type) on the film thickness (a) and process temperature (b).

decrease of the adhesion due to the increase of the stresses in the film. Maximum adhesion of the films is obtained for the thicknesses of $0.3-1.0 \mu \mathrm{m}$.

Appearance of chemical bonds between nickel and thermoelectric materials during the initial stage of the film formation increases the adhesion. Increase of the process temperature intensifies interaction between nickel and components of the thermoelectric material, which leads to the increase of the transition layer thickness, formed by the nickel compounds. At temperatures higher than $470 \mathrm{~K}$ (Fig. 2b) the size of the transition layer decreases the mechanical strength of the contact. Following increase of the process temperature up to $510 \mathrm{~K}$ decreases adhesion strength of the nickel film to the materials of $n$ and $p$-types by 11 and $23 \%$, respectively. Investigations have shown that optimal temperature regime of nickel film fabrication by electron beam evaporation, leading to the maximal adhesion of the formed contact to the materials of $n$ - and $p$-types, are $450-470$ and $380-400 \mathrm{~K}$, respectively.

\section{Conclusions}

Results of investigations allowed to optimize the technological process of the formation of ohmic contacts to the low temperature thermoelectric materials $\mathrm{Bi}_{2} \mathrm{Te}_{2.8} \mathrm{Se}_{0.2}$ ( $n$-type) and $\mathrm{Bi}_{0.5} \mathrm{Sb}_{1.5} \mathrm{Te}_{3}$ ( $p$-type), which has sufficiently increased effectiveness of thermoelectric devices.

Methods of vacuum deposition of nickel were used for the fabrication of ohmic contacts to the thermoelectric materials. Factors determining adhesion strength and resistivity of formed contacts were found. Technology of surface preparation for thermoelectric materials before the deposition of ohmic contacts was proposed, which consists of electrochemical polishing, ultrasound treatment, finish cleaning in toluene and isopropyl alcohol vapor, and annealing in vacuum. It was found that contacts formed using the electron-beam evaporation of nickel, possess maximal adhesion $\left(18-19 \mathrm{~N} / \mathrm{mm}^{2}\right)$. It was determined that high adhesion strength is caused by the existence of transition layer in the contact range of metal-thermoelectric material, formed due to the interaction of the metal with the components of thermoelectric material.

Results of the adhesion strength investigations allowed to determine optimal thicknesses of metal films $(0.5-1.0 \mu \mathrm{m})$ and temperature regime of metallization: 470 and $400 \mathrm{~K}$ for $n$ - and $p$-type materials, respectively. Proposed technology allows to obtain ohmic contacts with the resistance of the unit area not exceeding $10^{-10} \mathrm{Ohm} \mathrm{m}^{2}$.

\section{Acknowledgments}

This work was supported by the Ministry of Education and Science of Russian Federation project № 14.578.21.0038 (RFMEFI57814X0038).

\section{References}

[1] W. Heywang, Amorphe und Polykristalline Halbleiter, Halbleiter-Elektronik, Vol. 18, Springer Berlin Heidelberg, 1984, p. 252.

[2] Yu.I. Stern, M.Yu. Shtern, A.A. Sherchenkov, Russ. Microelectron. 41, 393 (2012).

[3] A.J. Kinlok, Adhesion and Adhesives, Springer Netherlands, 1987, p. 441.

[4] S.R. Morrison, The Chemical Physics of Surfaces, Springer US, 1990, p. 438.

[5] S.M. Sze, Physics of Semiconductor Devices, 2006, p. 832 . 